\title{
Psoas muscle index at the fifth lumbar vertebra as a predictor of survival in epithelial ovarian cancers
}

\author{
TOMOYUKI YOSHIKAWA ${ }^{1,2}$, MORIKAZU MIYAMOTO ${ }^{3}$, TADASHI AOYAMA ${ }^{3}$, HIROKO MATSUURA ${ }^{3}$, \\ HIDEKI IWAHASHI $^{3}$, HIROKI ISHIBASHI ${ }^{3}$, SOICHIRO KAKIMOTO ${ }^{3}$, TAKAHIRO SAKAMOTO ${ }^{3}$, \\ KAZUKI TAKASAKI ${ }^{3}$, JIN SUMINOKURA ${ }^{3}$, HITOSHI TSUDA ${ }^{4}$, \\ HIROYUKI KAWAGUCHI $^{2}$, AIHIDE YOSHINO ${ }^{1}$ and MASASHI TAKANO ${ }^{3}$ \\ ${ }^{1}$ Division of Palliative Care; ${ }^{2}$ Department of Clinical Oncology, National Defense Medical College Hospital; \\ ${ }^{3}$ Department of Obstetrics and Gynecology; ${ }^{4}$ Department of Basic Pathology, National Defense Medical College, \\ Tokorozawa, Saitama 359-8513, Japan
}

Received February 16, 2021; Accepted May 14, 2021

DOI: $10.3892 / \mathrm{mco} .2021 .2339$

\begin{abstract}
Almost a quarter of a century has passed since the term sarcopenia was defined. Sarcopenia is recognized as a poor prognostic factor in a variety of cancer types. In ovarian cancer, it remains controversial whether sarcopenia affects prognosis and how it should be evaluated. The present study aimed to evaluate the association between the volume of the psoas major muscle and survival in patients with epithelial ovarian cancer. Medical charts of patients with epithelial ovarian cancer who received first-line chemotherapy with paclitaxel and carboplatin at the National Defense Medical College Hospital (Tokorozawa, Japan) between April 2010 and January 2015 were retrospectively reviewed. The bilateral psoas major muscle areas at the fifth lumbar vertebra were measured using computed tomography images. The Institutional Review Board at National Defense Medical College Hospital (Tokorozawa, Japan) approved the study protocol. A total of 72 patients with epithelial ovarian cancer who received combination therapy with paclitaxel and carboplatin were identified and enrolled. The median psoas muscle index (PMI; psoas muscle major cross-sectional area divided by height squared) was $5.4 \mathrm{~cm}^{2} / \mathrm{m}^{2}$ (range, 3.3-10.0). Patients with higher PMI had significantly improved overall survival (OS) compared with those with lower PMI [log-rank test $\mathrm{P}=0.014$; hazard ratio (HR), 2.61; 95\% confidence interval (CI), 1.21-6.06]. Multivariate analysis for OS revealed that lower PMI was an independent unfavorable
\end{abstract}

Correspondence to: Dr Tomoyuki Yoshikawa, Division of Palliative Care, National Defense Medical College Hospital, 3-2, namiki, Tokorozawa, Saitama 359-8513, Japan

E-mail: tyoshi0204@ndmc.ac.jp

Key words: sarcopenia, skeletal muscle, psoas muscle, ovarian cancer, survival prognostic factor (HR, 3.87; 95\% CI, 1.37-12.1; $\mathrm{P}=0.0098)$. The volume of psoas major muscle mass could be a potential biomarker for prognosis in patients with epithelial ovarian cancer.

\section{Introduction}

In 1993, 'sarcopenia' was coined by Evans and Campbell (1) from a combination of the Greek 'sarx' for flesh and 'penia' for loss (2). The term sarcopenia, which was used for age-related loss of skeletal muscle mass and function, has also been adapted to refer to the severe loss of muscle mass and function associated with adverse outcomes in oncology $(3,4)$. In the clinical definition of cancer cachexia, sarcopenia is part of the diagnostic criteria along with weight loss and low Body mass index (BMI) (5). In clinical practice, ovarian cancers are often diagnosed at advanced stages, complicated by large tumors, massive ascites, and comorbidities, such as deep vein thrombosis (DVT) and pulmonary embolism (PE). As a result, many patients with ovarian cancers experience cachexia and sarcopenia because of appetite loss and systemic inflammation $(6,7)$.

Several reports, in which digital axial computed tomography (CT) was used, have suggested that sarcopenia is associated with poor prognoses in several solid cancers, including ovarian cancers $(8,9)$. In those papers, sarcopenia was assessed by the total area of the skeletal muscle mass at the third lumbar vertebra, which was demarcated using specialized imaging software that analyzed the number of Hounsfield units (HU) in the CT scans $(8,9)$. On the other hand, Rutten et al (10) reported that the psoas muscle area at the third lumbar vertebra was not representative of the total skeletal muscle area for the assessment of sarcopenia in ovarian cancer.

The purpose of this study is to evaluate the association between the prognoses of epithelial ovarian cancer patients and sarcopenia assessed based on the psoas major muscle area at the fifth lumbar vertebra, which was easily detected relative to the position of the ilium and sacrum (11). 


\section{Materials and methods}

Patients. After the institutional review board of the National Defense Medical College approved the study protocol of the present retrospective analyses, patients with ovarian cancers who met the inclusion criteria were enrolled. Comprehensive informed consent to use medical records had been obtained from each patient at the time of primary treatment. After IRB approval, the notice of the protocol including the use patients' records were open to the public, without any objection or rejection. So, all the records of the patients were used in the present study. The inclusion criteria were met for the following: (a) Patients with pathologically confirmed epithelial ovarian carcinoma who received primary therapy at the National Defense Medical College Hospital between April 2010 and January 2015, (b) patients who received combination chemotherapy using paclitaxel (PTX) and carboplatin (CBDCA; TC) as primary chemotherapy, (c) patients who had been scheduled to receive at least six cycles of combination chemotherapy using TC, (d) patients whose CT scan images of the abdomen and pelvis prior to chemotherapy were available, and (e) patients whose medical information was available (11).

A total of 72 patients were identified and enrolled in the present study. Of the patients in our previous study, excluded from the present study were those with a component of sarcoma or germ cell tumor (11). The median age of all patients was 62 years (range, 33-81 years), and the median follow-up duration was 49.5 months (range, 2-106 months). The number of patients according to the International Federation of Obstetrics and Gynecology (FIGO) classification (12) was as follows: 20 in stage I, 12 in stage II, 29 in stage III, and 11 in stage IV.

Measurement and definitions. We used the psoas muscle index (PMI $\left[\mathrm{cm}^{2} / \mathrm{m}^{2}\right]$; psoas muscle major cross-sectional area divided by height squared), as previously described (11). The areas of the right and left psoas major muscles at the fifth lumbar vertebra were measured using digital axial CT images, and these areas were calculated using the elliptical region of interest (ROI) with the picture archiving and communication system SYNAPSE (Fujifilm Corporation; Fig. 1).

Data collection. The other clinical variables assessed in this study were the following: Age, Eastern Cooperative Oncology Group (ECOG) performance status (PS) (https://ecog-acrin. org/resources/ecog-performance-status), FIGO stage (12), body mass index (BMI), serum albumin level, estimated creatinine clearance calculated using the Cockcroft-Gault equation just before chemotherapy, presence of massive ascites, comorbidities of DVT and PE, and type of chemotherapy (adjuvant or neoadjuvant chemotherapy), as previously described (11).

Treatment. In all cases, primary chemotherapy was combination therapy using the TC regimen. The TC regimen consisted of $175 \mathrm{mg} / \mathrm{m}^{2}$ of paclitaxel and area under the curve (AUC)=five to six of carboplatin on day one, repeated every three to four weeks. The relative dose intensity (RDI) was separately calculated for paclitaxel and carboplatin. We defined the standard $\mathrm{TC}$ regimen for RDI as $175 \mathrm{mg} / \mathrm{m}^{2}$ of paclitaxel and AUC=five of carboplatin on day one every three weeks.
Statistical analysis. Statistical analyses were performed using JMP Pro version 14 (SAS Institution Inc.). Overall survival (OS) was defined as the interval between the initial treatment and death. Serum tumor markers, including CA125, were not used for the definition of disease progression. Comparisons were evaluated using the chi-square test or the Fisher's exact probability test when appropriate. OS curves were generated using the Kaplan-Meier method. The comparison of the survival distributions was made using a log-rank test. Cox's proportional hazards model was used for univariate and multivariate analysis. Values of $\mathrm{P}<0.05$ were considered significant.

\section{Results}

Patient characteristics. A total of 72 patients with epithelial ovarian carcinomas were identified. The median psoas muscle index (PMI) was $5.39 \mathrm{~cm}^{2} / \mathrm{m}^{2}$ (range, 3.26-9.99). All of the patients were divided into two groups according to PMI value and further analyzed. The patients with lower PMI (PMI $<5.4 \mathrm{~cm}^{2} / \mathrm{m}^{2}$ ) were significantly associated with advanced stage $(\mathrm{P}=0.013)$, upfront chemotherapy $(\mathrm{P}=0.0007)$, histological type (Adeno, NOS; $\mathrm{P}=0.007$ ), hypoalbuminemia (serum albumin $<3.0 \mathrm{~g} / \mathrm{dl} ; \mathrm{P}<0.0001$ ), and massive ascites $(\mathrm{P}=0.002$; Table I).

Survival analyses. The patients with higher PMI had significantly improved OS compared with those with lower PMI (log-rank test $\mathrm{P}=0.014$; hazard ratio (HR), 2.61; 95\% confidence interval (CI), 1.21-6.06; Fig. 2). In the univariate analyses for OS, 11 variables reached statistical significance (BMI, ECOG PS, FIGO stage, histological type, initial therapy, residual disease status, PTX RDI, CBDCA RDI, serum albumin, massive ascites, and PMI; Table II). We investigated the multivariate analyses for OS with two patterns. In multivariate analysis \#1 for OS, all 11 explanatory variables were induced. In multivariate analysis \#2 for OS, four factors that were not known risk factors were excluded because of sample size, so 7 of 11 explanatory variables were induced. Lower PMI was identified as an independent unfavorable prognostic factor for OS in both multivariate analysis \#1 (HR, 3.83; 95\% CI, 1.29-13.0; $\mathrm{P}=0.015)$ and multivariate analysis \#2 (HR, 3.87; 95\% CI, 1.37-12.1; P=0.0098; Table II). Additionally, histological type was a significant independent prognostic factor in both multivariate analyses \#1 and \#2. Moreover, BMI $\left(\geq 25 \mathrm{~kg} / \mathrm{m}^{2}\right)$ and residual disease status before chemotherapy was a significant independent poor prognostic factor in multivariate analysis \#2 (Table II).

\section{Discussion}

In the present study, the PMI evaluated at the fifth lumbar vertebra as an index for sarcopenia was identified as an independent unfavorable prognostic factor in Japanese patients who received upfront treatment for ovarian cancers. To our knowledge, this is the first report using multivariate analyses to demonstrate the association between the loss of psoas major muscle mass and risk of poor outcome in ovarian cancer patients who received TC therapy. This finding provides 


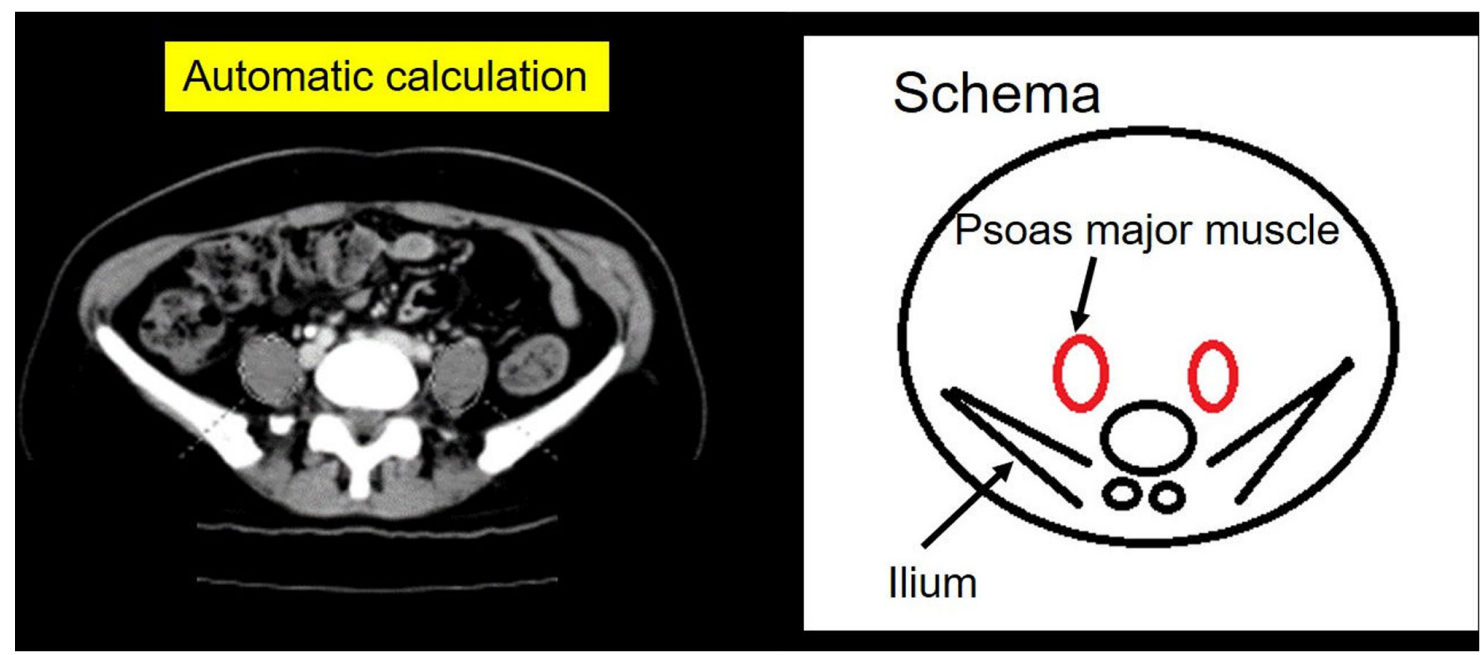

Figure 1. Representative image of an axial CT scan for the evaluation of the psoas muscle index. The areas of the right and left psoas major muscles at the fifth lumbar vertebra were calculated using the elliptical region of interest with SYNAPSE (10). It is easy to measure the psoas major muscle areas when the fifth lumbar vertebra, where the ilium bones are located in the lower half dorsal side in the horizontal section of CT imaging, is used as a landmark.

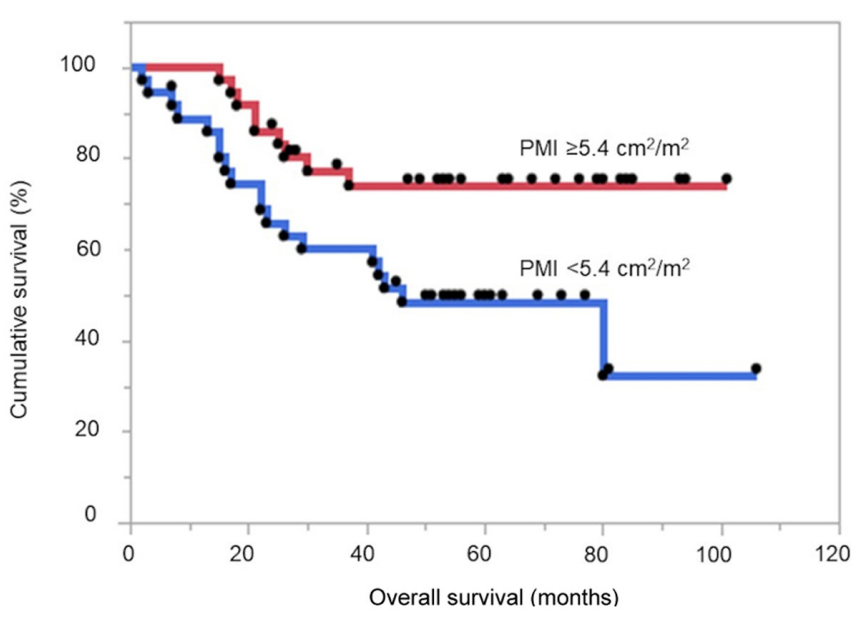

Figure 2. OS curves of patients with epithelial ovarian cancer according to PMI values. Patients with lower PMI had significantly poorer OS compared with the cases with higher PMI (log-rank test $\mathrm{P}=0.014$ ). Red line, cases with PMI $\geq 5.4 \mathrm{~cm}^{2} / \mathrm{m}^{2}$; blue line, cases with PMI $<5.4 \mathrm{~cm}^{2} / \mathrm{m}^{2}$. OS, overall survival; PMI, psoas muscle index.

evidence that we might be able to evaluate only one muscle to determine the prognosis of epithelial ovarian cancer in primary therapy using the TC regimen. PMI was associated with overall survival in ovarian cancer patients, and the significant impact of PMI on prognosis was confirmed by two independent multivariate analyses.

Conrad et al (13) reported that pre-operative PMI at the fourth lumbar vertebra was not associated with poor prognosis and that PMI, in combination with hypoalbuminemia, was associated with unfavorable prognosis in advanced ovarian cancer. In their paper, the mean PMI was $2.80 \mathrm{~cm}^{2} / \mathrm{m}^{2}$. On the other hand, in this study, the mean PMI was $5.48 \mathrm{~cm}^{2} / \mathrm{m}^{2}$. We speculate that the difference in muscle volume distribution due to the range of stages had a strong influence on survival.

Rutten et al (10) insisted that the change in psoas muscle area was not representative of the change in total muscle area and should not be used as a substitute for the total skeletal muscle in predicting survival in patients with ovarian cancer. In their study, the median psoas muscle area at the third lumbar vertebra was $13.3 \mathrm{~cm}^{2}$. On the other hand, in this study, the median psoas muscle area at the fifth lumbar vertebra was $12.7 \mathrm{~cm}^{2}$. We speculated that our patients had a smaller muscle mass because the fifth lumbar vertebra has a larger muscle area than the third lumbar vertebra (Fig. 3). In our study, the proportion of overweight and obese patients (18.1\%) was small compared to the proportion (35.7\%) in Rutten's report. As shown in our previous study, there is a positive and moderate correlation between BMI and PMI, which might have influenced the different results between their study and ours (11).

The psoas area is often measured at a standard lumbar vertebral landmark (L3 or L4), but sometimes, unreliable soft tissue landmarks, such as the umbilicus, have been used (14). Previous papers mainly reported on sarcopenia assessed by the total area of skeletal muscle mass at the third lumbar vertebra $(3,6,8-10)$. It is easy to measure the psoas major muscle areas when the fifth lumbar vertebra, where the ilium bones are located in the lower half dorsal side in the horizontal section of CT imaging, is used as a landmark. When evaluating sarcopenia using the psoas major muscle only, the belly of the muscle is not located at the third lumbar vertebra but actually at the fifth lumbar vertebra (Fig. 3). In this study, we used a PMI calculated using the elliptical region of interest that could be obtained with a simple method, as previously described (11). We believe that easier detection and measurement of PMI are of great importance for clinical practice.

In a recent meta-analysis not including Asian countries, the skeletal mass index was significantly associated with survival (15). Conversely, in a cohort study from the United States of America, sarcopenia was not associated with poor survival outcomes in epithelial ovarian cancer patients receiving upfront platinum and taxane-based chemotherapy (16). In a report from Asia, Kim et al (17) found that the skeletal mass index was not associated with worse survival 
Table I. Characteristics of the patients according to psoas muscle index.

\begin{tabular}{|c|c|c|c|}
\hline \multirow[b]{2}{*}{ Characteristics } & \multicolumn{2}{|c|}{ Psoas muscle index } & \multirow[b]{2}{*}{ P-value } \\
\hline & High $(n=36)$ & Low $(n=36)$ & \\
\hline Age, years & & & 0.10 \\
\hline Median & 60 & 65 & \\
\hline Range & $33-78$ & $41-81$ & \\
\hline$\geq 70, \mathrm{n}(\%)$ & $7(19.4)$ & $10(27.8)$ & \\
\hline$<70, \mathrm{n}(\%)$ & $29(80.6)$ & $26(72.2)$ & \\
\hline BMI, n (\%) & & & 0.06 \\
\hline$\geq 25 \mathrm{~kg} / \mathrm{m}^{2}$ & $10(27.8)$ & $3(8.3)$ & \\
\hline$<25 \mathrm{~kg} / \mathrm{m}^{2}$ & $26(72.2)$ & $33(91.7)$ & \\
\hline ECOG performance status, n (\%) & & & 0.13 \\
\hline 0 & $29(80.6)$ & $21(58.3)$ & \\
\hline 1 & $6(16.7)$ & $11(30.6)$ & \\
\hline 2 & $1(2.8)$ & $4(11.1)$ & \\
\hline FIGO stage, n (\%) & & & 0.01 \\
\hline I & $15(41.7)$ & $5(13.9)$ & \\
\hline II & $7(19.4)$ & $5(13.9)$ & \\
\hline III & $12(33.3)$ & $17(47.2)$ & \\
\hline IV & $2(5.6)$ & $9(25.0)$ & \\
\hline Histological type, n (\%) & & & $<0.01$ \\
\hline Serous & $9(25.0)$ & $20(55.6)$ & \\
\hline Endometrioid & $6(16.7)$ & $5(13.9)$ & \\
\hline Clear cell & $11(30.6)$ & $5(13.9)$ & \\
\hline Mucinous & $2(5.6)$ & $0(0.0)$ & \\
\hline Mixed & $4(11.1)$ & $0(0.0)$ & \\
\hline Others & $2(5.6)$ & $0(0.0)$ & \\
\hline Adeno, NOS & $2(5.6)$ & $6(16.7)$ & \\
\hline Initial therapy, n (\%) & & & $<0.01$ \\
\hline PDS & $28(77.8)$ & $13(36.1)$ & \\
\hline Chemotherapy & $8(22.2)$ & $23(63.9)$ & \\
\hline Residual disease at PDS, n (\%) & & & 0.89 \\
\hline Complete & $21(58.3)$ & $10(27.8)$ & \\
\hline Optimal or Suboptimal & 7 (19.4) & $3(8.3)$ & \\
\hline Upfront chemotherapy, n (\%) & & & 0.27 \\
\hline NAC followed by IDS & $6(16.7)$ & $21(58.3)$ & \\
\hline Induction chemotherapy alone & $2(5.6)$ & $2(5.6)$ & \\
\hline PTX RDI, \% & & & 0.63 \\
\hline Median & 70.6 & 68.7 & \\
\hline$\geq 70, \mathrm{n}(\%)$ & $20(55.6)$ & $17(47.2)$ & \\
\hline$<70, \mathrm{n}(\%)$ & $16(44.4)$ & $19(52.8)$ & \\
\hline CBDCA RDI, $\%$ & & & 0.63 \\
\hline Median & 72.9 & 71.0 & \\
\hline$\geq 70, \mathrm{n}(\%)$ & $22(61.1)$ & $19(52.8)$ & \\
\hline$<70, \mathrm{n}(\%)$ & $14(38.9)$ & $17(47.2)$ & \\
\hline Serum albumin, $\mathrm{n}(\%)$ & & & $<0.01$ \\
\hline$\geq 3.0 \mathrm{~g} / \mathrm{dl}$ & $34(94.4)$ & $19(52.8)$ & \\
\hline$<3.0 \mathrm{~g} / \mathrm{dl}$ & $2(5.6)$ & $17(47.2)$ & \\
\hline Ccr, n $(\%)$ & & & 0.71 \\
\hline$\geq 60 \mathrm{ml} / \mathrm{min}$ & $33(91.7)$ & $31(86.1)$ & \\
\hline$<60 \mathrm{ml} / \mathrm{min}$ & $3(8.3)$ & $6(16.7)$ & \\
\hline
\end{tabular}


Table I. Continued.

Psoas muscle index

\begin{tabular}{lcc} 
Characteristics & High $(\mathrm{n}=36)$ & Low $(\mathrm{n}=36)$ \\
\hline Massive ascites, $\mathrm{n}(\%)$ & & $18(50.0)$ \\
Yes $(>1,000 \mathrm{ml})$ & $5(13.9)$ & $18(50.0)$ \\
No & $31(86.1)$ & \\
PE or DVT, n $(\%)$ & $3(8.3)$ & $6(16.7)$ \\
Yes & $33(91.7)$ & $30(83.3)$ \\
No & & 0.48 \\
\hline
\end{tabular}

ECOG, Eastern Cooperative Oncology Group; FIGO, the International Federation of Gynecology and Obstetrics; PDS, primary debulking surgery; NAC, neoadjuvant chemotherapy; IDS, interval debulking surgery; RDI, relative dose index; Ccr, estimated creatinine clearance; PE, pulmonary embolism; DVT, deep vein thrombosis.

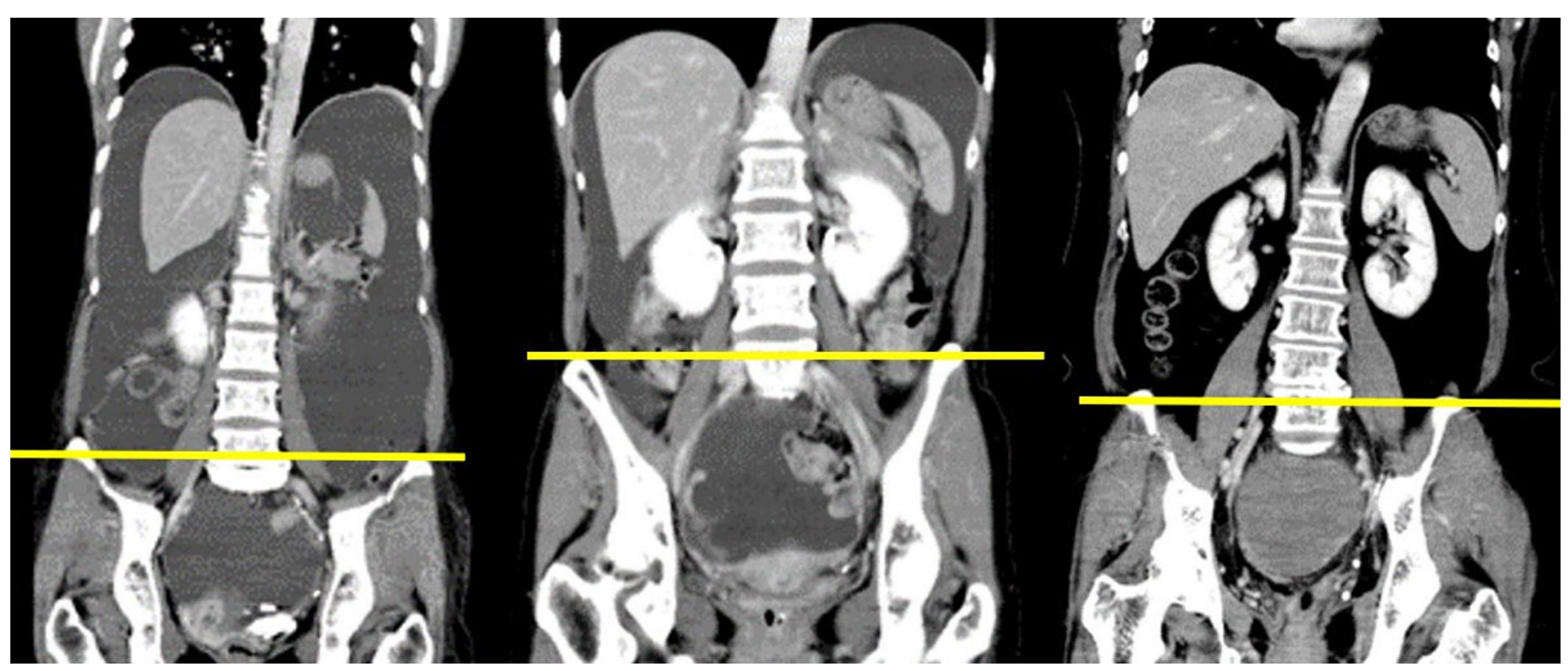

Figure 3. Images of coronal CT for the evaluation of the psoas muscle areas of 3 patients with ovarian cancer. When evaluating sarcopenia using only the psoas major muscle, the belly of the muscle is not located at the third lumbar vertebra but at the fifth lumbar vertebra. The yellow line represents the level of the fifth lumbar spine.

outcomes, but patients with a high fat-to-muscle ratio showed significantly worse OS in advanced-stage, high-grade serous ovarian carcinoma. Conversely, Huang et al (18) reported that the skeletal muscle index was independently associated with poor OS in patients with stage III epithelial ovarian cancer treated with primary debulking surgery and adjuvant platinum-based chemotherapy. Although sarcopenia has been recognized as an important prognostic factor, sarcopenia remains controversial in terms of what its optimal index is and what the optimal cut-off values are. Moreover, values will differ according to the number of lumbar vertebra and the patient's race.

In pancreatic cancer, sarcopenic obesity has been recognized as a poor prognostic factor in meta-analysis (19). In ovarian cancer, a Korean group reported that a high fat-to-muscle ratio was related to significantly worse OS (17). Furthermore, in the present study, being overweight (BMI $\geq 25 \mathrm{~kg} / \mathrm{m}^{2}$ ) was a poor prognostic factor, independent of sarcopenia, for OS in multivariate analysis \#2. These results are congruent with those reported by Kim et al (17). Sarcopenic obesity is associated with elevated markers of inflammation such as interleikin-6 (IL-6), tumor necrosis factor (TNF) and C-reactive protein (CRP) in postmenopausal women (20). Inflammation that causes cachexia and sarcopenia causes sarcopenic obesity, and obesity itself has a negative effect on inflammation and tumor-promoting effects (21). We believe that sarcopenic obesity potentially has a significant impact on survival, even in ovarian cancer.

In conclusion, sarcopenia assessed using PMI values measured at the fifth lumbar vertebra had a significant and independent impact on OS in patients with epithelial ovarian cancers, though further analyses including a large number of patients are needed. Moreover, the PMI value should be evaluated along with impacts of other drugs, such as molecularly targeted drugs and immune checkpoint inhibitors. The measurement of the psoas major muscle areas at the fifth lumbar vertebra could be an important indicator for the prognosis of epithelial ovarian cancers. 
Table II. Cox univariate and multivariate analyses for overall survival.

\begin{tabular}{|c|c|c|c|c|c|c|}
\hline \multirow[b]{2}{*}{ Variables } & \multicolumn{2}{|c|}{ Univariate analysis } & \multicolumn{2}{|c|}{ Multivariate analysis \#1 } & \multicolumn{2}{|c|}{ Multivariate analysis \#2 } \\
\hline & $\mathrm{HR}(95 \% \mathrm{CI})$ & P-value & $\mathrm{HR}(95 \% \mathrm{CI})$ & P-value & HR $(95 \% \mathrm{CI})$ & P-value \\
\hline Age, years & & 0.45 & & & & \\
\hline$<70$ & 1 & & & & & \\
\hline$\geq 70$ & $1.38(0.57-3.03)$ & & & & & \\
\hline $\mathrm{BMI}, \mathrm{kg} / \mathrm{m}^{2}$ & & 0.04 & & 0.10 & & 0.04 \\
\hline$<25$ & 1 & & 1 & & 1 & \\
\hline$\geq 25$ & $2.52(1.03-5.58)$ & & $2.88(0.81-10.0)$ & & $3.37(1.05-10.5)$ & \\
\hline ECOG performance status & & 0.03 & & 0.72 & & 0.68 \\
\hline 0 & 1 & & 1 & & 1 & \\
\hline 1,2 & $2.37(1.09-4.99)$ & & $0.82(0.27-2.43)$ & & $1.21(0.48-3.10)$ & \\
\hline FIGO stage & & $<0.01$ & & 0.13 & & 0.39 \\
\hline I, II & 1 & & 1 & & 1 & \\
\hline III, IV & $3.14(1.39-7.99)$ & & $2.49(0.76-8.11)$ & & $1.72(0.48-3.09)$ & \\
\hline Histological type & & $<0.01$ & & 0.04 & & $<0.01$ \\
\hline Adeno, NOS & $7.78(2.95-18.5)$ & & $3.20(1.04-9.89)$ & & $5.15(1.75-14.6)$ & \\
\hline Others & 1 & & 1 & & 1 & \\
\hline Initial therapy & & $<0.01$ & & 0.89 & & 0.12 \\
\hline Surgery & 1 & & 1 & & 1 & \\
\hline Chemotherapy & $3.01(1.42-6.65)$ & & $0.89(0.13-5.20)$ & & $0.32(0.08-1.35)$ & \\
\hline $\begin{array}{l}\text { Residual disease status } \\
\text { before chemotherapy }\end{array}$ & & $<0.01$ & & 0.22 & & 0.03 \\
\hline None & 1 & & 1 & & 1 & \\
\hline Others & $5.31(2.18-15.9)$ & & $2.25(0.60-8.87)$ & & $4.04(1.14-14.9)$ & \\
\hline PTX RDI, \% & & $<0.01$ & & 0.10 & & \\
\hline$\geq 70$ & 1 & & 1 & & & \\
\hline$<70$ & $4.97(2.20-12.7)$ & & $4.51(0.70-25.1)$ & & & \\
\hline CBDCA RDI, $\%$ & & $<0.01$ & & 0.91 & & \\
\hline$\geq 70$ & 1 & & 1 & & & \\
\hline$<70$ & $3.56(1.67-8.09)$ & & $1.09(0.24-6.55)$ & & & \\
\hline Serum albumin, g/dl & & $<0.05$ & & & & \\
\hline$\geq 3.0$ & 1 & & 1 & 0.29 & & \\
\hline$<3.0$ & $2.30(1.01-4.91)$ & & $0.45(0.11-2.05)$ & & & \\
\hline $\mathrm{Ccr}, \mathrm{ml} / \mathrm{min}$ & & 0.65 & & & & \\
\hline$\geq 60$ & 1 & & & & & \\
\hline$<60$ & $1.29(0.38-3.34)$ & & & & & \\
\hline Massive ascites $(\geq 1,000 \mathrm{ml})$ & & 0.02 & & 0.70 & & \\
\hline No & 1 & & 1 & & & \\
\hline Yes & $2.44(1.12-5.14)$ & & $1.37(0.28-7.36)$ & & & \\
\hline PE or DVT & & 0.39 & & & & \\
\hline No & 1 & & & & & \\
\hline Yes & $1.63(0.47-4.26)$ & & & & & \\
\hline Psoas muscle index & & $<0.01$ & & $<0.01$ & & $<0.01$ \\
\hline $\operatorname{High}\left(\geq 5.4 \mathrm{~cm}^{2} / \mathrm{m}^{2}\right)$ & 1 & & 1 & & 1 & \\
\hline Low $\left(<5.4 \mathrm{~cm}^{2} / \mathrm{m}^{2}\right)$ & $2.61(1.21-6.06)$ & & $3.83(1.29-13.0)$ & & $3.87(1.37-12.1)$ & \\
\hline
\end{tabular}

In multivariate analysis \#1 for OS, all 11 explanatory variables were induced. In multivariate analysis \#2 for OS, four factors that were not known risk factors were excluded because of the sample size, so 7 of 11 explanatory variables were included. ECOG, Eastern Cooperative Oncology Group; FIGO, the International Federation of Gynecology and Obstetrics; RDI, relative dose index; Ccr, estimated creatinine clearance; PE, pulmonary embolism; DVT, deep vein thrombosis. 


\section{Acknowledgements}

The authors would like to thank Ms. Hiromi Kubota (Department of Clinical Oncology, National Defense Medical College Hospital, Tokorozawa, Japan) and Ms. Aya Yokoyama (Tokyo, Japan) for continuous contribution to the present clinical study.

\section{Funding}

No funding was received.

\section{Availability of data and materials}

The datasets used and/or analysed during the current study are available from the corresponding author on reasonable request.

\section{Authors' contributions}

TY and MT conceived the study. TY, MM, TA, HM, HIw, HIs, SK, TS, KT, JS, HT, HK, AY and MT designed the analysis. TY, MM, TA, HM, HIw, HIs, SK, TS, KT and JS collected and interpreted the patient data. TY, MM, HT and MT confirmed the authenticity of the raw data. TY, MM and TA analysed the data. TY, HT, HK, AY and MT wrote the draft of manuscript. TY, MM, HT, HK, AY and MT critically revised the manuscript critically for important intellectual content. TY, HT, HK, AY and MT provided final approval of the version to be published. All authors read and approved the final manuscript.

\section{Ethics approval and consent to participate}

The Institutional Review Board at National Defense Medical College Hospital (Saitama, Japan) approved this study (approval no. 4021), which proceeded in accordance with the ethical standards established in the Declaration of Helsinki in 1995 (Brazil 2013 revision). The comprehensive written informed consent to use medical records had been obtained from each patient at the time of primary treatment. Consent from the participants was obtained using the opt-out principle. The nature of the study and the right of refusal to participate were disclosed to the public online.

\section{Patient consent for publication}

Not applicable.

\section{Competing interests}

The authors declare that they have no competing interests.

\section{References}

1. Evans WJ and Campbell WW: Sarcopenia and age-related changes in body composition and functional capacity. J Nutr 123 (Suppl 2): S465-S468, 1993.

2. Ryan AM, Power DG, Daly L, Cushen SJ, Ní Bhuachalla $\overline{\mathrm{E}}$ and Prado CM: Cancer-associated malnutrition, cachexia and sarcopenia: The skeleton in the hospital closet 40 years later. Proc Nutr Soc 75: 199-211, 2016.
3. Baumgartner RN, Koehler KM, Gallagher D, Romero L, Heymsfield SB, Ross RR, Garry PJ and Lindeman RD: Epidemiology of sarcopenia among the elderly in New Mexico. Am J Epidemiol 147: 755-763, 1998.

4. Shachar SS, Williams GR, Muss HB and Nishijima TF: Prognostic value of sarcopenia in adults with solid tumours: A meta-analysis and systematic review. Eur J Cancer 57: 58-67, 2016.

5. Fearon K, Strasser F, Anker SD, Bosaeus I, Bruera E, Fainsinger RL, Jatoi A, Loprinzi C, MacDonald N, Mantovani G, et al: Definition and classification of cancer cachexia: An international consensus. Lancet Oncol 12: 489-495, 2011.

6. Solheim TS, Fayers PM, Fladvad T, Tan B, Skorpen F, Fearon K, Baracos VE, Klepstad P, Strasser F and Kaasa S; European Palliative Care Research Collaborative (EPCRC) and the European Pharmacogenetic Study (EPOS): Is there a genetic cause of appetite loss?-an explorative study in 1,853 cancer patients. J Cachexia Sarcopenia Muscle 3: 191-198, 2012.

7. Feliciano EM, Kroenke CH, Meyerhardt JA, Prado CM, Bradshaw PT, Kwan ML, Xiao J, Alexeeff S, Corley D, Weltzien E, et al: Association of systemic inflammation and sarcopenia with survival in nonmetastatic colorectal cancer: Results from the C SCANS study. JAMA Oncol 3: e172319, 2017.

8. Rutten IJ, van Dijk DP, Kruitwagen RF, Beets-Tan RG, Olde Damink SW and van Gorp T: Loss of skeletal muscle during neoadjuvant chemotherapy is related to decreased survival in ovarian cancer patients. J Cachexia Sarcopenia Muscle 7: 458-466, 2016.

9. Kumar A, Moynagh MR, Multinu F, Cliby WA, McGree ME, Weaver AL, Young PM, Bakkum-Gamez JN, Langstraat CL, Dowdy SC, et al: Muscle composition measured by CT scan is a measurable predictor of overall survival in advanced ovarian cancer. Gynecol Oncol 142: 311-316, 2016.

10. Rutten IJ, Ubachs J, Kruitwagen RF, Beets-Tan RG, Olde Damink SW and Van Gorp T: Psoas muscle area is not representative of total skeletal muscle area in the assessment of sarcopenia in ovarian cancer. J Cachexia Sarcopenia Muscle 8: 630-638, 2017.

11. Yoshikawa T, Takano M, Miyamoto M, Yajima I, Shimizu Y, Aizawa Y, Suguchi Y, Moriiwa M, Aoyama T, Soyama H, et al: Psoas muscle volume as a predictor of peripheral neurotoxicity induced by primary chemotherapy in ovarian cancers. Cancer Chemother Pharmacol 80: 555-561, 2017.

12. Javadi S, Ganeshan DM, Qayyum A, Iyer RB and Bhosale P: Ovarian cancer, the revised FIGO staging system, and the role of imaging. AJR Am J Roentgenol 206: 1351-1360, 2016.

13. Conrad LB, Awdeh H, Acosta-Torres S, Conrad SA, Bailey AA, Miller DS and Lea JS: Pre-operative core muscle index in combination with hypoalbuminemia is associated with poor prognosis in advanced ovarian cancer. J Surg Oncol 117: 1020-1028, 2018.

14. Baracos VE: Psoas as a sentinel muscle for sarcopenia: A flawed premise. J Cachexia Sarcopenia Muscle 8: 527-528, 2017.

15. Ubachs J,Ziemons J, Minis-Rutten IJ, Kruitwagen RF, Kleijnen J, Lambrechts S, Olde Damink SW, Rensen SS and Van Gorp T: Sarcopenia and ovarian cancer survival: A systematic review and meta-analysis. J Cachexia Sarcopenia Muscle 10: 1165-1174, 2019.

16. Staley SA, Tucker K, Newton M, Ertel M, Oldan J, Doherty I, West L, Zhang Y and Gehrig PA: Sarcopenia as a predictor of survival and chemotoxicity in patients with epithelial ovarian cancer receiving platinum and taxane-based chemotherapy. Gynecol Oncol 156: 695-700, 2020.

17. Kim SI, Kim TM, Lee M, Kim HS, Chung HH, Cho JY and Song YS: Impact of CT-determined sarcopenia and body composition on survival outcome in patients with advanced-stage high-grade serous ovarian carcinoma. Cancers (Basel) 12: 559, 2020.

18. Huang CY, Yang YC, Chen TC, Chen JR, Chen YJ, Wu MH, Jan YT, Chang CL and Lee J: Muscle loss during primary debulking surgery and chemotherapy predicts poor survival in advanced-stage ovarian cancer. J Cachexia Sarcopenia Muscle 11: 534-546, 2020.

19. Mintziras I, Miligkos M, Wächter S, Manoharan J, Maurer E and Bartsch DK: Sarcopenia and sarcopenic obesity are significantly associated with poorer overall survival in patients with pancreatic cancer: Systematic review and meta-analysis. Int J Surg 59: 19-26, 2018.

20. Dutra MT, Avelar BP, Souza VC, Bottaro M, Oliveira RJ, Nóbrega OT and Moreno Lima R: Relationship between sarcopenic obesity-related phenotypes and inflammatory markers in postmenopausal women. Clin Physiol Funct Imaging 37: 205-210, 2017.

21. Iyengar NM, Gucalp A, Dannenberg AJ and Hudis CA: Obesity and cancer mechanisms: Tumor microenvironment and inflammation. J Clin Oncol 34: 4270-4276, 2016.

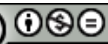

This work is licensed under a Creative Commons Attribution-NonCommercial-NoDerivatives 4.0 International (CC BY-NC-ND 4.0) License. 